\title{
Strain characteristics of cotton yarns depending on the strain rate and methods of their manufacture
}

\author{
Bakhodir Baymuratov ${ }^{*}$, Shamsidin Tulanov ${ }^{1,2}$, Karim Sultanov $^{1,2}$, and Sabida Ismailova ${ }^{1}$ \\ ${ }^{1}$ Tashkent Institute of Textile and Light Industry, 100100 Tashkemt, Uzbekistan \\ ${ }^{2}$ Institute of Mechanics and Seismic Stability of Structures of the Academy of Sciences of the \\ Republic of Uzbekistan, 100128 Tashkent, Uzbekistan
}

\begin{abstract}
One of the main reasons for the nonlinearity of the tension diagrams of cotton yarns is the variability of their moduli of elasticity and plasticity under strain. The changes in strain moduli obtained from the tensile diagrams confirm this. The strain curve has ten parameters, the values of which depend on the method of yarn manufacture and the strain rate. Based on the results of processing the tension diagrams of cotton yarn, obtained by carded and combed systems by ring and rotor spinning (CD-carded ring-spun, OE-carded rotor-spun, CM-combed ring-spun), at strain rates from $0,0033 \mathrm{~s}-1$ to $0,033 \mathrm{~s}-1$, the values of these ten parameters were determined and analyzed. Their quantitative and qualitative dependence on the method of their manufacture and strain rate are shown.
\end{abstract}

\section{Introduction}

Experimental determination of the strength of cotton yarns, a decently time-consuming and time-consuming problem. Determining the strength of yarns at high speeds of their movement or at high speeds of their deformation is a complex experimental problem, so the strength of yarns is determined, evaluated and predicted by formulas developed based on the analysis of experimental results [1]. These formulas are called empirical. Currently, there are no empirical formulas for determining the strength of yarns, depending on their types or the method of production [2].

The solution of suitable problems of textile threads and yarns during their movement in the technological processes of textile production requires knowledge of the law of their deformation. As reported, the real mathematical model or the law of deformation is nonlinear [3]. The nonlinearity of the yarn deformation law is suspended by the replacement of it is structures during stretching. This is an experimentally proven fact even in classical works [4], it is necessary to load it into the laws of deformation of cotton yarn. This path led to the development of a physical nonlinear elastic-viscoplastic law of deformation of cotton yarn in [5].

\footnotetext{
* Corresponding author: bakhodir_b@mail.ru
} 
The nonlinear strain of textile cotton yarns (hereinafter referred to as yarn) under stretching to breakage has attracted the attention of researchers for a long time [6]. The manifestation of the nonlinear properties of the yarn was explained by the change in strain modulus of the yarn under its stretching. This concept was developed in [7]. Indeed, when the yarn is stretched, its fibrous structure undergoes changes. There occurs a mutual relative mixing of fibers and changes in the structure of the yarn appear at the beginning of strain. Consequently, the mechanical properties of the yarn change as well. The regularities of changes in mechanical characteristics of carded rotor spun yarn are discussed in detail in [8]. The results of experiments on stretching yarns to breakage, for three types of yarns, at different rates of stretching are given in [9].

\section{Materials and Methods}

In [9], the yarns produced by carded and combed systems by ring and rotor methods with a linear density $T=29.0$ tex were used in experiments. The speed of their motion varied within the limits of the capability of the "Statimat C" tensile installation from $v=100$ $\mathrm{mm} / \mathrm{min}$ to $1000 \mathrm{~mm} / \mathrm{min}$. The strain rate, at a base yarn length $L_{0}=500 \mathrm{~mm}$, varied from $\varepsilon_{v}=0,0033 \mathrm{~s}^{-1}$ to $\varepsilon_{v}=0,033 \mathrm{~s}^{-1}$. Since these values of the strain rate are close to the static stretching of the yarn, the stretching process is considered quasi-static. It is shown in [5-8] that when the yarn is stretched to breakage, in the process of yarn strain all stages of strain are manifested - elastic, visco-elastic, elastic-viscoplastic strain. Therefore, the general concept of strain modulus is introduced, which includes elastic, viscous, plastic moduli. This strain modulus is actually a quasi-static modulus $E_{S}$.

\section{Results and Discussion}

In [5-8] it is shown that in the process of yarn stretching, the strain modulus, depending on the strain itself, changes nonlinearly. A nonlinear function $E_{S}(\varepsilon)$ characterizing changes in strain modulus of the yarn is shown in Fig. 1, for a combed ring yarn with a linear density $T=29.0$ tex, obtained by stretching it to breakage at a speed of $1000 \mathrm{~mm} / \mathrm{min}$, determined from the diagram $F(\varepsilon)$ according to the methods described in [4-6].

The curve in Fig. 1 is the secant modulus of strain when the yarn is stretched from the initial value $\varepsilon=0$ to breakage $\varepsilon=\varepsilon_{k}$. Curve 1, as seen from Fig. 1, has ten characteristic values for five points $\varepsilon_{N} E_{N}, \varepsilon_{e} E_{e}, \varepsilon_{m} E_{m}, \varepsilon_{c} E_{c}, \varepsilon_{k} E_{k}$. The strain modulus $E_{S}$ for $\varepsilon=\varepsilon_{N} \approx 0$ has an initial value of $E_{N}$.

At the beginning of the stretching process, the strain modulus $E_{s}$ begins to decrease to a value of $E_{v}$ in the interval $\varepsilon_{N} \varepsilon_{e}$. Then the value of $E_{s}$ increases to the value of $E_{m}$ in the interval $\varepsilon_{e} \varepsilon_{m}$. In some cases, for example for carded rotor-spun yarns, after the modulus $E_{s}$ reaches the value of $E_{m}$, there is a fall down. The strain modulus $E_{s}=E_{c}$ at $\varepsilon=\varepsilon_{c}$ is intermediate, at $E_{s}=E_{k}$ and $\varepsilon=\varepsilon_{k}$, the yarn breaks. For any trajectories of strain change, curve 1 in Fig. 1 can be analytically described [7,8] with nine parameters of yarn strain $E_{N}, E_{e}, E_{m}, E_{c}, E_{k}, \varepsilon_{e}, \varepsilon_{m}, \varepsilon_{c}, \varepsilon_{k}\left(\varepsilon_{N}=0\right)$; and intermediate values of the strain modulus are determined at any point on Curve 1 (Fig. 1), which are necessary to define the yarn strength according to the method described in [5]. 


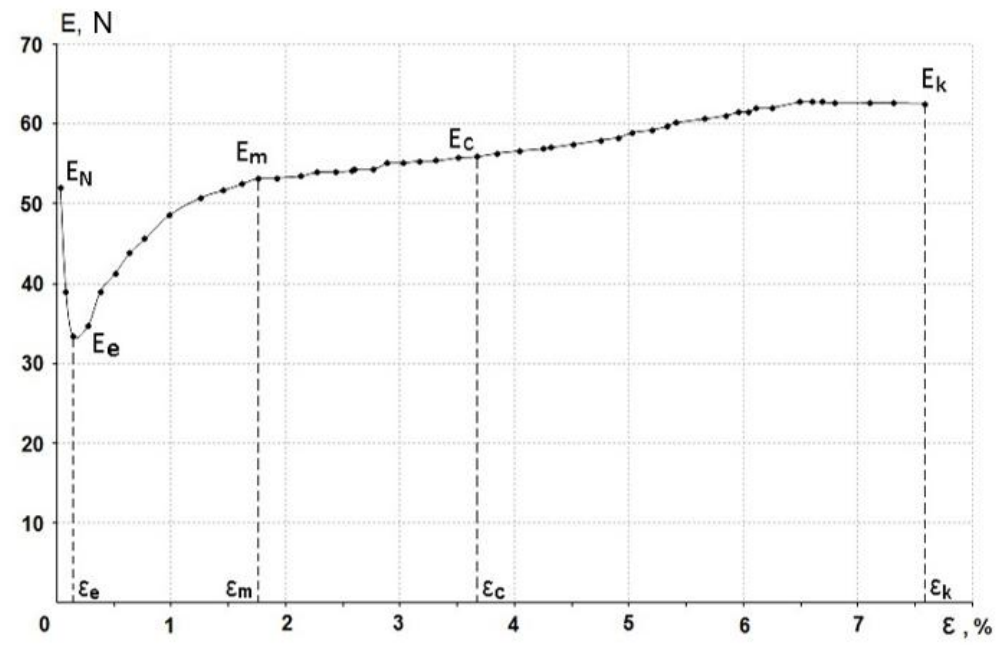

Fig. 1. Curve $E_{s}(\varepsilon)$ for combed ring yarns at $v=1000 \mathrm{~mm} / \mathrm{min}$

The values of strain parameters $E(\varepsilon)$ are determined from the graphs automatically using a special program. When the cursor is set at any point of Curve 1, the values of the coordinate of this point, $\varepsilon_{i}$ and $E_{i}$, appear on the monitor screen. The values of $\varepsilon_{i}$ and $E_{i}$ are equal to the values of strain parameters at this point, respectively. The values of the strain moduli $E_{N}, E_{e}, E_{m}, E_{c}, E_{k}$ are in $N$ (Newton or $\mathrm{MPa}$ ), and the values of $\varepsilon_{e}, \varepsilon_{m}, \varepsilon_{c}, \varepsilon_{k}$-are dimensionless. The change in parameters $E_{N}, E_{e}, E_{m}, E_{c}, E_{k}, \varepsilon_{e}, \varepsilon_{m}, \varepsilon_{c}, \varepsilon_{k}$ depending on the strain rate for the above types of yarn was determined for ten values of strain rate.

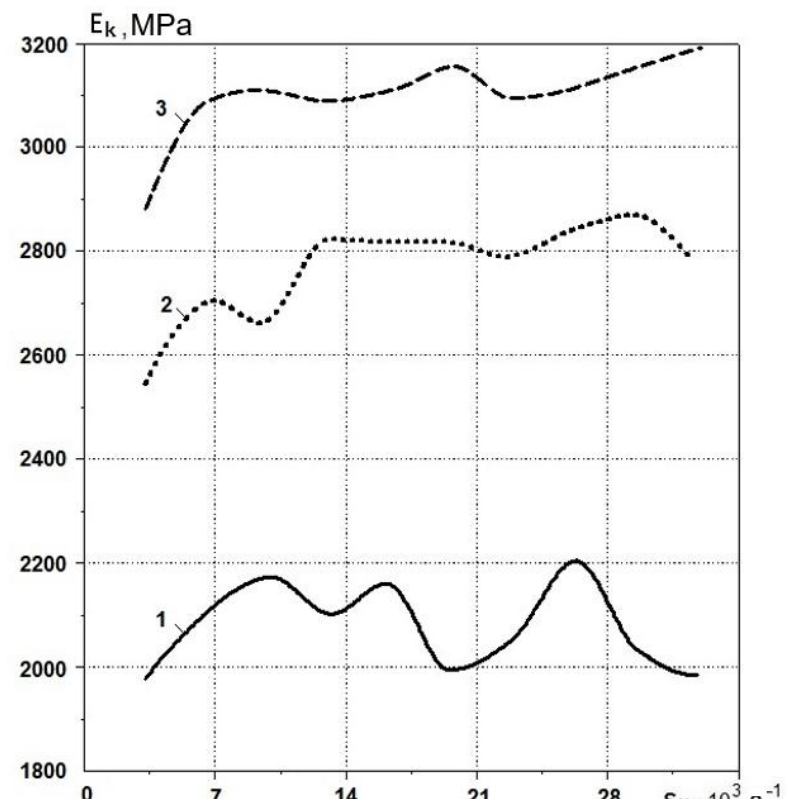

Fig. 2. Changes in strain parameter $E_{k}$ depending on the strain rate: 1 - carded rotor-spun yarn; 2 carded ring-spun yarn; 3 - combed ring-spun yarn. 
Fig. 2 shows changes in parameter $E_{k}$ of the function $E(\varepsilon)$ when the speed $v$ changes from $100 \mathrm{~mm} / \mathrm{min}$ to $1000 \mathrm{~mm} / \mathrm{min}$, which corresponds to a change in strain rate from $\varepsilon_{v}=$ $0,0033 \mathrm{~s}^{-1}$ to $\varepsilon_{v}=0,033 \mathrm{~s}^{-1}$. Here, solid curve 1 refers to carded rotor yarn, dashed-line curve 2 refers to carded ring yarn, dotted curve 3 refers to combed ring yarn.

In the case of carded rotor yarns (curve 1), the value of $E_{k}$ fluctuates between $E_{k}=2000$ $\mathrm{MPa}$ and $E_{k}=2200 \mathrm{MPa}$ with an increase in strain rate. The value of $E_{k}$ in the case of curves 2 and 3 first increases, and then, starting from the strain rate value of $\varepsilon_{v}=0,014 \mathrm{~s}^{-1}$, it remains approximately constant at $E_{k}=2800 \mathrm{MPa}$ and $E_{k}=3100 \mathrm{MPa}$.

Changes in parameters $E_{S}(\varepsilon)$ show that in different methods of cotton yarns manufacture, the values of these parameters are different. With an increase in strain rate from $\varepsilon_{v}=0,0033 \mathrm{~s}^{-1}$ to $\varepsilon_{v}=0,033 \mathrm{~s}^{-1}$, the values of parameters $E_{N}, E_{e}, E_{m}, E_{c}, E_{k}$ remain constant on average. A pattern similar to the one in Fig. 2 is observed for the remaining parameters of strain modulus.

Fig. 3 shows the changes in strain parameters $\varepsilon_{e}, \varepsilon_{m}, \varepsilon_{c}, \varepsilon_{k}$. With an increase in strain rate from $\varepsilon_{v}=0,0033 \mathrm{~s}^{-1}$ to $\varepsilon_{v}=0,033 \mathrm{~s}^{-1}$, the values of these parameters remain constant on average. For different yarn production methods, the values of $\varepsilon_{e}$ are practically the same. The values of $\varepsilon_{m}, \varepsilon_{c}, \varepsilon_{k}$ slightly depend on the yarn production methods. To simplify the problem, the dependence of all parameters on the spinning methods is averaged over their values for all strain rates.

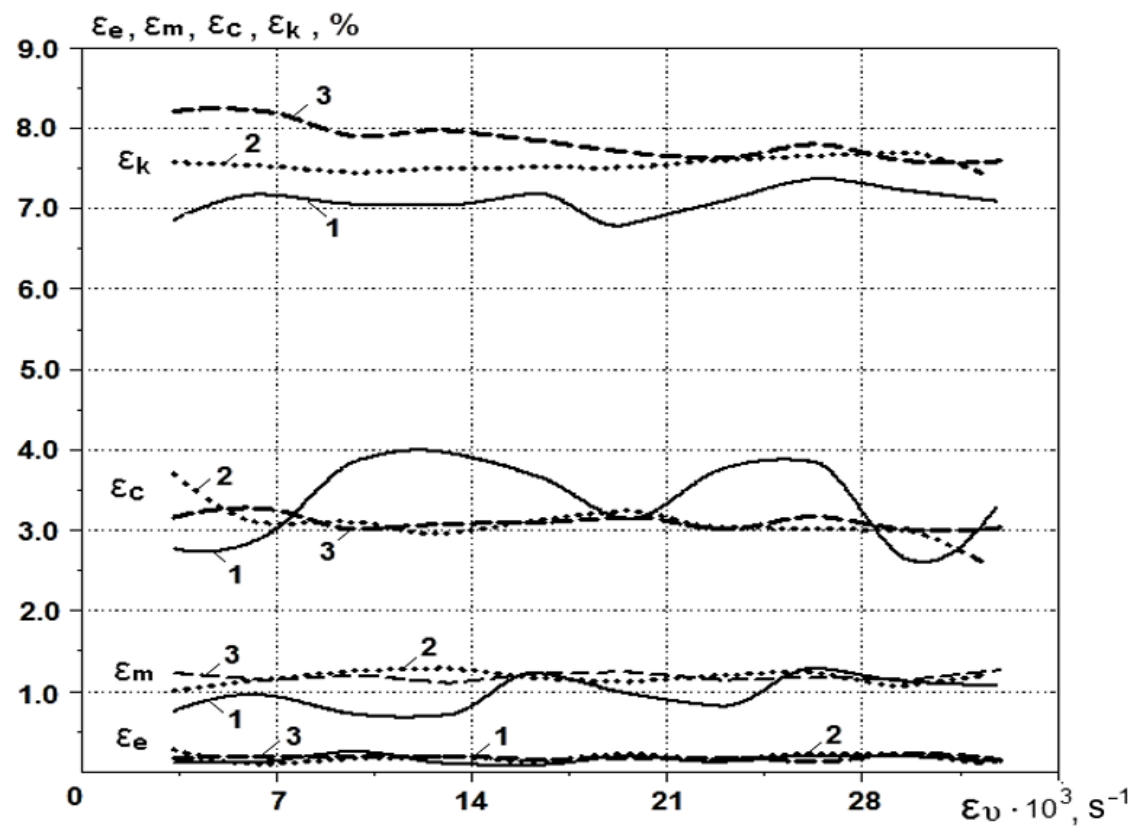

Fig. 3. Changes in strain parameters depending on the strain rate: 1 - carded rotor-spun yarn; 2 carded ring-spun yarn; 3 - combed ring-spun yarn

The averaged values of all 14 strain parameters according to the methods of their production are shown in Table 1 . Based on the data from Table 1, the differences in the values of the parameters are determined in percent and shown in Table 2. 
Table 1. Average values of strain parameters for different strain rates

\begin{tabular}{|c|c|c|c|}
\hline \multirow[b]{2}{*}{$\begin{array}{c}\text { Strain } \\
\text { parameters }\end{array}$} & \multicolumn{3}{|c|}{ Methods of cotton yarn manufacture } \\
\hline & $\begin{array}{c}\text { Carded rotor- } \\
\text { spun yarns }\end{array}$ & $\begin{array}{l}\text { Carded ring- } \\
\text { spun yarns }\end{array}$ & $\begin{array}{c}\text { Combed ring-spun } \\
\text { yarns }\end{array}$ \\
\hline$E_{N}, \mathrm{~N}$ & 42.70 & 41.6 & 51.31 \\
\hline$E_{N}, \mathrm{MPa}$ & 2138.34 & 2115.61 & 2618.01 \\
\hline$\varepsilon_{e}$ & 0.00144 & 0.00175 & 0.00170 \\
\hline$E_{e}, \mathrm{~N}$ & 31.88 & 27.63 & 33.65 \\
\hline$E_{e}, \mathrm{MPa}$ & 1626.55 & 1410.01 & 1717.14 \\
\hline$\varepsilon_{m}$ & 0.00958 & 0.01165 & 0.01177 \\
\hline$E_{m}, \mathrm{~N}$ & 42.62 & 42.56 & 47.01 \\
\hline$E_{m}, \mathrm{MPa}$ & 2174.62 & 2171.50 & 2397.24 \\
\hline$\varepsilon_{c}$ & 0.0338 & 0.0310 & 0.0311 \\
\hline$E_{c}, \mathrm{~N}$ & 40.64 & 46.35 & 51.14 \\
\hline$E_{c}, \mathrm{MPa}$ & 2073.50 & 2365.01 & 2609.44 \\
\hline$\varepsilon_{k}$ & 0.0710 & 0.07546 & 0.0784 \\
\hline$E_{k}, \mathrm{~N}$ & 40.73 & 54.22 & 60.75 \\
\hline$E_{k}, \mathrm{MPa}$ & 2078.06 & 2766.41 & 3099.30 \\
\hline
\end{tabular}

Table 2 shows a comparative assessment of cotton yarns manufactured by three spinning systems used in experiments, where number 1 corresponds to carded rotor yarn, 2 - to carded ring yarn and 3 - to combed ring yarn. The values of the averaged strain parameters were compared for these types of yarns.

It can be clearly seen that the yarn produced by the combed system in the ring-spun method is the strongest yarn. The values of the strain parameters of carded ring yarn are 5$33 \%$ greater in terms of strain moduli $E$ compared to carded rotor-spun yarns, and, with the exception of values $\varepsilon_{e}$ and $\varepsilon_{c}$, are from 8 to $18 \%$ greater in terms of strain $\varepsilon$. The strain parameters of the combed ring yarn are also from 10 to $20 \%$ greater in terms of strain moduli $E$ and for carded ring-spun yarns are from 0 to $4 \%$ greater in terms of strain $\varepsilon$. The values of strain parameters of the carded ring yarn are $20 \%$, greater in terms of strain modulus $E$ and for the carded rotor yarn are from 6 to $18 \%$ greater in terms of strain $\varepsilon$.

The above results show that the strain parameters of the yarns, characterizing the nonlinear dependence $E_{S}(\varepsilon)$, within the considered limits of the change in strain rate, practically do not depend on the strain rate. However, the parameter values $E_{N}, E_{e}, E_{m}, E_{c}, E_{k}, \varepsilon_{e}, \varepsilon_{m}, \varepsilon_{c}, \varepsilon_{k}$ up to $20-30 \%$ depend on the yarn production methods. This means that when predicting the strength of yarns produced by different methods, this circumstance must be taken into account. 
Table 2. Differences in the values of strain parameters for different methods of yarn manufacture

\begin{tabular}{|c|c|c|c|}
\hline \multirow{3}{*}{$\begin{array}{c}\text { Strain } \\
\text { parameters }\end{array}$} & \multicolumn{3}{|c|}{ Methods of cotton yarn manufacture } \\
\hline & $\begin{array}{c}\text { Carded rotor-spun } \\
\text { yarns }\end{array}$ & $\begin{array}{c}\text { Carded ring-spun } \\
\text { yarns }\end{array}$ & $\begin{array}{c}\text { Combed ring-spun } \\
\text { yarns }\end{array}$ \\
\hline & $\begin{array}{c}1 \text { and } 2 \\
\%\end{array}$ & $\begin{array}{c}1 \text { and } 3 \\
\%\end{array}$ & $\begin{array}{c}2 \text { and } 3 \\
\%\end{array}$ \\
\hline$E_{N}, \mathrm{~N}$ & 2.89 & 16.8 & 19.19 \\
\hline$E_{N}, \mathrm{MPa}$ & 1.06 & 18.32 & 19.19 \\
\hline$\varepsilon_{e}$ & 17.7 & 15.3 & 2.9 \\
\hline$E_{e}, \mathrm{~N}$ & 13.3 & 5.27 & 18.0 \\
\hline$E_{e}, \mathrm{MPa}$ & 13.3 & 5.27 & 18.0 \\
\hline$\varepsilon_{m}$ & 17.8 & 18.6 & 1.02 \\
\hline$E_{m}, \mathrm{~N}$ & 0.15 & 9.3 & 9.45 \\
\hline$E_{m}, \mathrm{MPa}$ & 0,15 & 9.3 & 9.42 \\
\hline$\varepsilon_{c}$ & 8.3 & 8.3 & 0 \\
\hline$E_{c}, \mathrm{~N}$ & 12.3 & 20.54 & 9.4 \\
\hline$E_{c}, \mathrm{MPa}$ & 12.3 & 20.54 & 9.4 \\
\hline$\varepsilon_{k}$ & 5.9 & 9.4 & 3.75 \\
\hline$E_{k}, \mathrm{~N}$ & 24.9 & 33.0 & 10.8 \\
\hline$E_{k}, \mathrm{MPa}$ & 24.9 & 33.0 & 10.8 \\
\hline
\end{tabular}

\section{Conclusions}

1. Based on the analysis of the experimental results, the values of strain parameters for different strain rates of yarns manufactured by three technological methods were determined.

2. The influence of the yarn strain rate on the values of the yarn strain parameters was determined. It was established that the strain rate of the yarn under tension, within the variation limits considered in the experiments, has little effect on the values of strain parameters.

3. The influence of yarn production methods on the strain characteristics of yarns was determined. The influence of technological methods of yarn production on the values of strain moduli $E_{S}$ (up to $30 \%$ ), and on the values of strain parameters $\varepsilon$ (up to $20 \%$ ) was established.

\section{References}

1. V. Shcherbakov, Applied and structural mechanics of fibrous materials, 304 (Tisoprint Press, Moscow, 2013) 
2. W. Morton, J. Hearle, Physical properties of textile fibers, 765 (Cambridge Press, London, 2008)

3. I. Migushov, The mechanics of textile yarn and fabric, 160 (Light industry Press, Moscow, 1980)

4. S. Ismailova, K. Sultanov, Mechanics of Solids 5, 578-592 (2015)

5. K. Sultanov, S. Ismoilova, Journal of Fashion Technology \& Textile Engineering 10(3), 11 (2017)

6. K. Sultanov, S. Ismoilova, N. Mamatova, Journal of Fashion Technology \& Textile Engineering 6(1), 10 (2018)

7. K. Sultanov, S. Ismailova, Sh. Tulanov, Izvestiya Vuzov. Technology of the textile industry 5(365), 109-115 (2016)

8. S. Ismailova, K. Sultanov, Sh. Tulanov, Izvestiya Vuzov. Technology of the textile industry 4 (364), 63-67 (2016)

9. K. Sultanov, S. Ismailova, Izvestiya Vuzov. Technology of the textile industry 3(375), 95-101 (2018) 